\title{
Trehalose synthesis in Aspergillus niger: characterization of six homologous genes, all with conserved orthologs in related species
}

\author{
Åsa Svanström ${ }^{1}$ Martin Richard van Leeuwen ${ }^{2}$, Jan Dijksterhuis ${ }^{2}$ and Petter Melin ${ }^{1,3^{*}}$
}

\begin{abstract}
Background: The disaccharide trehalose is a major component of fungal spores and is released upon germination. Moreover, the sugar is well known for is protective functions, e.g. against thermal stress and dehydration. The properties and synthesis of trehalose have been well investigated in the bakers' yeast Saccharomyces cerevisiae. In filamentous fungi, such knowledge is limited, although several gene products have been identified.
\end{abstract}

Results: Using Aspergillus niger as a model fungus, the aim of this study was to provide an overview of all genes involved in trehalose synthesis. This fungus has three potential trehalose-6-phosphate synthase encoding genes, tpsA-C, and three putative trehalose phosphate phosphatase encoding genes, tppA-C, of which two have not previously been identified. Expression of all six genes was confirmed using real-time PCR, and conserved orthologs could be identified in related Aspergilli. Using a two-hybrid approach, there is a strong indication that four of the proteins physically interact, as has previously been shown in $S$. cerevisiae. When creating null mutants of all the six genes, three of them, $\Delta \operatorname{tps} A, \Delta \operatorname{tpp} A$ and $\triangle t p p B$, had lower internal trehalose contents. The only mutant with a pronounced morphological difference was $\triangle t p p A$, in which sporulation was severely reduced with abnormal conidiophores. This was also the only mutant with accumulated levels of trehalose-6-phosphate, indicating that the encoded protein is the main phosphatase under normal conditions. Besides $\triangle t p p A$, the most studied deletion mutant in this work was $\triangle t p p B$. This gene encodes a protein conserved in filamentous Ascomycota. The $\triangle t p p B$ mutant displayed a low, but not depleted, internal trehalose content, and conidia were more susceptible to thermal stress.

Conclusion: A. niger contains at least 6 genes putatively involved in trehalose synthesis. Gene expressions related to germination have been quantified and deletion mutants characterized: Mutants lacking tps $A$, tppA or tppB have reduced internal trehalose contents. Furthermore, tppA, under normal conditions, encodes the functional trehalose-6-phosphate-phosphatase.

Keywords: Ascomycota, Conidia, Germination, Saccharomyces cerevisiae, Stress-resistance, Targeted gene deletion

\section{Background}

Trehalose ( $\alpha$-D-glucopyranosyl- $\alpha$-D-glucopyranoside) is a non-reducing disaccharide that is present in a wide variety of organisms. It has been isolated from plants, fungi, nematodes and insects [1-3]. In fungi, trehalose has been shown to accumulate in dispersal and survival structures such as spores (where it can constitute as

\footnotetext{
* Correspondence: petter.melin@icloud.com

'Uppsala BioCenter, Department of Microbiology, Swedish University of Agricultural Sciences, P.O. Box 7025, SE-750 07 Uppsala, Sweden

${ }^{3}$ Present address: Swedish Chemicals Agency, Box 2, SE-172 13 Sundbyberg, Sweden

Full list of author information is available at the end of the article
}

much as $10 \%$ of the dry weight), sclerotia, and in yeast cells going into stationary phase $[3,4]$. Since the sugar is rapidly degraded when these structures germinate or resume vegetative growth, early research concluded that trehalose serves as a storage molecule [5,6]. However, later studies showed that the function of trehalose is more complex and diverse than just serving as an energy reserve; the molecule has been shown to function as a regulator of carbon metabolism [1], a signaling molecule and a protection molecule against various kinds of abiotic stress [3,7]. Several fungal species have been shown to induce trehalose production as a stress response. Examples include: Saccharomyces cerevisiae [8,9], Zygosaccharomyces 
bailii [10], A. nidulans [11], A. fumigatus [12], Rhizopus oryzae [13], and Botrytis cinerea [14]. Trehalose is known to protect both proteins and lipid membranes of living cells against stressors such as heat, desiccation and cold. Although the mode of bio-protection of trehalose is not fully elucidated, three main hypotheses are generally accepted, and the true mechanism is likely a combination of these. The hypotheses include: water replacement (direct interaction of trehalose with the protected structure through hydrogen bonds); mechanical entrapment (glass formation of trehalose that creates a protective coating around the structure); preferential exclusion (bulk water is ordered around trehalose and is thereby separated from the bio-molecule, which then becomes more compact and stabilized) $[15,16]$. The physico-chemical properties of trehalose that lie behind these hypotheses include several crystalline forms, a high glass transition temperature, and the stereochemistry of the sugar $[7,15]$.

In fungi, trehalose is synthesized via the intermediate trehalose-6-phosphate (T6P) and involves two enzymatic steps. First, T6P is formed from one glucose-6-phosphate and one UDP-glucose catalyzed by T6P-synthase (here called TPS). In the next step, the phosphate molecule is removed by trehalose-phosphate-phosphatase (here called TPP) yielding trehalose $[1,11]$. The organism in which trehalose synthesis has been most thoroughly studied is $S$. cerevisiae. Here, four homologous gene products responsible for trehalose synthesis physically interact forming a "trehalose synthase complex", which consists of one TPS (called Tps1), one TPP (called Tps2), and two other subunits, Tsl1 and Tps3, with proposed regulatory and stabilizing functions [6,17-19]. In filamentous fungi, the gene products involved in trehalose synthesis are not as thoroughly investigated as in $S$. cerevisiae, but have been studied with respect to germination [20], plant pathology [21] and human pathology [12,22].

Within Aspergilli, several individual gene products have been identified and characterized. In $A$. niger, two Tps1 orthologs, tps $A$ and tps $B$, have been identified and characterized. At ambient temperature, the trehalose level of $\triangle t p s A$ mycelia was lowered compared to wildtype. In contrast to the constitutively expressed $t p s A$, the expression of $\operatorname{tps} B$ was induced by thermal stress [23]. In the opportunistic human pathogen $A$. fumigatus, four Tps1 paralogs, tps $A-D$ have been identified [12]. When deleting these genes, the authors found that either tps $A$ or tps $B$ was sufficient to maintain normal trehalose levels, but if both genes were deleted, the resulting mutant strain was depleted of trehalose and showed slower germination rates as well as higher susceptibility to heat and oxidative stress compared to wild-type. Another notable finding was that this double mutant was hypervirulent in infected mice [12]. In A. nidulans, a Tps1 ortholog, tpsA, has been identified and deleted. In this mutant, trehalose was not accumulated, and in addition, the authors could conclude that in A. nidulans trehalose is important for resistance to continual exposure to sub-lethal stress but not to short exposure of lethal stress [11]. In contrast to S. cerevisiae, tps mutants in Aspergilli are able to utilize glucose as carbon source $[11,23,24]$. All identified Tps1 orthologs in Aspergilli are generally much shorter than the $S$. cerevisiae Tps1, around 500 amino acids compared to 1447.

Besides Tps1 orthologs, two Tps2 orthologs have been identified within the Aspergilli, one in A. nidulans [25] and one in $A$. fumigatus [22]: In both species they are designated orlA. The $\triangle$ orlA mutant of $A$. fumigatus had a pronounced phenotype with abolished asexual reproduction as well as decreased virulence. However, the phenotype could be restored to wild-type appearance by growing the mutant on media containing an osmotic stabilizer (sorbitol or glycerol). As also observed in A. nidulans, the $A$. fumigatus $\triangle$ orlA mutant strain contained wild-type levels of trehalose but the T6P levels were elevated [22,25].

In this study we focused on trehalose synthesis in filamentous fungi, and more specifically, in Aspergillus niger. This is a common food spoilage mould as well as an industrially important organism, utilized for production of citric acid, for instance [26]. Six genes, tps $A$ (ANI_1_1406074), tpsB (ANI_1_1078064), tpsC (ANI_ 1_1216124), tppA (ANI_1_1432094), tppB (ANI_1_ 48114) and tppC (ANI_1_2070064) were identified to be involved in trehalose biosynthesis. Expression of these genes was studied during conidial outgrowth. In addition, we deleted these genes and characterized the mutants in terms of trehalose and T6P content, protein interactions, and stress survival coupled to situations often occurring in foodstuff.

\section{Methods}

Software, hardware and computer-based analyses used in this study

GraphPad Prism ${ }^{\circledast}$ version 5 was used for generating figures (line drawings) and calculating mean, standard error of the mean, and significance between samples (using one or two way ANOVA and Bonferroni post-test). Adobe Illustrator CS5 and Adobe Photoshop CS6 were used for managing pictures (cropping and minor changes in contrast levels for best visualization). Bio-Rad CFX $96^{\text {тм }}$ Real-Time System was used for generating gene expression data and the Bio-Rad CFX Manager ${ }^{\text {TM }}$ version 1.6 software was used for analyzing the data. MacVector version 12 was used for primer design and phylogenetic analyses.

\section{Culture maintenance, spore preparation and spore densities}

The fungal strains used in this study are listed in Table 1. As wild-type, we used the $A$. niger N402 strain, which is 
Table 1 Strains used in this study

\begin{tabular}{|c|c|c|}
\hline Strain & Genotype & Reference \\
\hline N402 & $\operatorname{cspAl}$ & {$[27]$} \\
\hline MA70.15 & 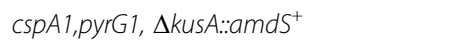 & {$[32]$} \\
\hline MA169.4 & 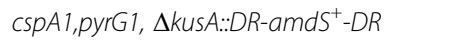 & {$[33]$} \\
\hline J699* ( $\operatorname{tps} A)$ & cspA1,pyrG1, $\Delta$ kusA::amdS ${ }^{+}, \Delta \operatorname{tps} A:$ :pyrG & This study \\
\hline $\mathrm{J} 700(\Delta \operatorname{tps} B)$ & cspA1,pyrG1, $\Delta$ kusA:::amdS ${ }^{+}, \Delta t p s B:: p y r G$ & This study \\
\hline J701 ( $\Delta \operatorname{tps} C)$ & cspA1,pyrG1, $\Delta t p s C:: p y r G$ & This study \\
\hline$J 684(\Delta t p p A)$ & cspA1,pyrG1, $\Delta$ kusA::amdS ${ }^{+}, \Delta t p p B:: p y r G$ & This study \\
\hline$J 685(\Delta t p p B)$ & cspA1,pyrG1, $\Delta$ kusA:::amdS ${ }^{+}, \Delta t p p B:: p y r G$ & This study \\
\hline $\mathrm{J} 702(\Delta t p p B 2)$ & cspA1,pyrG1, $\Delta$ tppB::pyrG & This study \\
\hline $\mathrm{J} 686(\Delta t p p C)$ & cspA1,pyrG1, $\Delta$ kusA::amdS ${ }^{+}, \Delta t p p C:: p y r G$ & This study \\
\hline J689 (pyrG+) & $\operatorname{cspA1}, \Delta k u s A:: a^{\prime} d S^{+}$ & {$[28]$} \\
\hline J693 (tppB+) & cspA1,pyrG1, $\Delta$ tppB:::pyrG, tppB::.hph & This study \\
\hline
\end{tabular}

*Strain numbers from the fungal collection at the Department of Microbiology, Swedish University of Agricultural Sciences.

also the mother strain of all generated mutants [27]. The strains were maintained on Aspergillus Minimal Media (AMM) as previously described [28]. For the MA70.15 and MA169.4 strains, AMM was supplemented with $10 \mathrm{mM}$ uridine. The complemented strain $(\operatorname{tpp} B+)$ was maintained on AMM containing Hygromycin B $(0.10 \mathrm{mg} / \mathrm{ml})$. The $\triangle t p p A$ mutant was tested for sporulation both on AMM agar and on AMM agar containing $1.2 \mathrm{M}$ sorbitol. Normally plates were incubated at $25^{\circ} \mathrm{C}$ for 14 days. All deletion mutants as well as the control strains were tested for growth in $10,15,20,25,30$ and $37^{\circ} \mathrm{C}$ for 14 days. For trehalose measurements, conidia were harvested from plates incubated at $25^{\circ} \mathrm{C}$ for $5,14,28$ and 90 days. Spore suspensions were prepared in water containing Tween $80(0.01 \% \mathrm{v} / \mathrm{v})$, were filtered through sterile Miracloth (Calbiochem), and the spore count was determined using a Bürker chamber. To estimate the number of conidia produced, a circular area of $95 \mathrm{~mm}^{2}$ was cut out from centrally inoculated AMM plates that had been incubated at $25^{\circ} \mathrm{C}$ for 14 days. $10 \mathrm{ml}$ of water containing Tween $80(0.01 \% \mathrm{v} / \mathrm{v})$ and 10 glass beads ( $2 \mathrm{~mm}$ in diameter) were added to the agar plug, the mixture was vortexed for $10 \mathrm{~min}$ and spore concentrations were counted in a Bürker chamber. Three biological replicates, each calculated from the average of three technical replicates, were used for all samples.

\section{Low-temperature scanning electron microscopy (SEM)}

Wild-type, N402, and $\Delta t p p A$ were grown for 1 week on AMM. Margins of colonies containing conidiophores were excised with a surgical blade and carefully transferred into a copper cup (diameter $10 \mathrm{~mm}$, height $8 \mathrm{~mm}$ ). Dislodging during snap freezing was prevented by gluing agar blocks in the copper cup with frozen tissue medium (KP-Cryoblock, Klinipath, Duiven, the Netherlands). The sample was snap-frozen in nitrogen slurry and immediately transferred in a vacuum transfer device to an Oxford CT1500 Cryostation attached to a JEOL 5600LV scanning electron microscope (JEOL, Tokyo, Japan). Electron micrographs were acquired from uncoated frozen samples, or after sputter-coating with gold three times during $30 \mathrm{~s}$. Micrographs of uncoated samples were taken at an acceleration voltage of $2.5 \mathrm{kV}$, and consisted of 30 averaged fast scans (SCAN 2 mode). Coated samples were observed at $5 \mathrm{kV}$ using F4 scans.

\section{Extraction of nucleic acids}

DNA was extracted as previously described [28]. RNA from dormant conidia and conidia in early stages of germination $(0$ and $3 \mathrm{~h})$ was extracted according to Leeuwen and co-workers [29]. RNA from germinating spores (6 and $12 \mathrm{~h}$ ), mycelia and sporulating mycelia (plate) were extracted according to Plumridge and co-workers [30]. As a final step in both protocols, the RNA products were purified using a Qiagen RNeasy Mini kit (RNA clean up protocol).

\section{Two-hybrid assay}

The two-hybrid assay was performed using the BACTH System Kit (Euromedex). Full-length cDNA for all six genes were amplified using primers with internal restriction sites (Table 2). After digestion of the PCR products, the inserts were ligated into linearized and dephosphorylated pKT25 and pUT18C vectors and used to transform $E$. coli. All ligations in this work were performed with the ReadyToGo ligation kit (GE Healthcare) and were transformed into NEB 10- $\beta$ Competent E. coli cells (New England Biolabs), unless otherwise stated. Correct insertions were confirmed with vector specific primers (Table 2) followed by sequencing. Successful clones were co-transformed into electrocompetent BTH101 cells and selected on LA plates supplemented with ampicillin $(100 \mu \mathrm{g} / \mathrm{ml})$ and kanamycin $(50 \mu \mathrm{g} / \mathrm{ml})$. The proteinprotein interactions were assayed according to the manufacturer's protocol with the following modifications. One fresh colony of each interaction was transferred to $100 \mathrm{ml}$ conical flasks with $5 \mathrm{ml} \mathrm{LB}$ supplemented with ampicillin $50 \mu \mathrm{g} / \mathrm{ml}$, kanamycin $50 \mu \mathrm{g} / \mathrm{ml}$ and $0.5 \mathrm{mM}$ IPTG, and incubated with shaking at $100 \mathrm{rpm}$ at $20^{\circ} \mathrm{C}$ for $72 \mathrm{~h}$. The extent of protein-protein interaction was measured with $\beta$-galactosidase assays as units/mg dry weight.

\section{CDNA synthesis and Real-Time PCR}

Using total RNA as template, cDNA was synthesized using a polyT primer (Table 2) and the enzyme SuperscriptIII (Invitrogen) according to the manufacturer's protocol. Quantitative real-time PCR was performed with the BioRad CFX-96 system using the EvaGreen 
Table 2 Primers used for CDNA synthesis, qPCR and Two-Hybrid cloning

\begin{tabular}{|c|c|c|}
\hline Primer name & Sequence 5'-3' & Purpose \\
\hline $\mathrm{T} 12 \mathrm{VN}$ & TTTTTITTTTVN & \multirow[t]{2}{*}{ cDNA synthesis } \\
\hline tpsAF & TGAGGGCTGTTGTGAATGAGC & \\
\hline tpsAR & ACTCGGAAAGCACCAAGACACC & qPCR tpsA \\
\hline $\operatorname{tpsBF}$ & GTGGGCAGAATCAACGGAAAG & \multirow{2}{*}{$\mathrm{qPCR}$ tps $B$} \\
\hline $\operatorname{tpsBR}$ & TGAACACTTGGATAGTTCGGCAAC & \\
\hline tpsCF & TTGCCGATGCCTGCTTGTTG & \multirow{2}{*}{ qPCR tpsC } \\
\hline $\operatorname{tps} C R$ & TTCGCTGGATGGAAAGTAAGACAC & \\
\hline tppAF & TTGAAGACACCGTTGGGAAGAG & \multirow{2}{*}{ qPCR tppA } \\
\hline tppAR & GGAGCAAAAGATGAACTCAGGAGC & \\
\hline tppBF & TGGACACTTACCTCTGGGATGAAG & \multirow{2}{*}{ qPCR tppB } \\
\hline tppBR & GCTGATGGGCATTGAGTATTTCC & \\
\hline tppCF & AAAGCCAAAGCAGCCGAATC & \multirow{2}{*}{ qPCR tppC } \\
\hline $\operatorname{tppCR}$ & TGCCCGTTAGTATCCTCAGCAGAG & \\
\hline actF & TCGTGACCTGACGGATTACCTC & \multirow{2}{*}{$\mathrm{qPCR}$ actin } \\
\hline actR & TGGAAGAAGGAGCAAGAGCAGTG & \\
\hline pKT25F & ACGATTTCGAGGCGGTCAAG & \multirow{2}{*}{ Confirmation of cloned cDNA to pKT25 vector } \\
\hline pKT25R & GATGTGCTGCAAGGCGATTAAG & \\
\hline pUT18CF & TGTCTTCTACGAGAACCGTGCATAC & \multirow{3}{*}{ Confirmation of cloned cDNA to pUT18C vector } \\
\hline pUT18CR & CGGTGAAAACCTCTGACACATGC & \\
\hline tpsAFpst & GACTCTGCAGCCGTTTCCGACAGCATGCCTT & \\
\hline tpsAFbam & TATCTGGATCCCGTTTCCGACAGCATGCCTT & \multirow[t]{2}{*}{ Cloning of tpsA cDNA } \\
\hline tpsARkpn & TGATCGGTACCAGCTCACTGTGCCACCTGCT & \\
\hline tpsBFbam & ATCAGGATCCTCCTTITCCAATGGCTGCCAA & \multirow{2}{*}{ Cloning of $\operatorname{tps} B$ cDNA } \\
\hline tpsBeco & ATCAGAATTCAGCTGCAGTCATAACATAATCA & \\
\hline tpsCFxba & AGTCTCTAGAATCCAGAATGACGAAGCGCAA & \multirow{2}{*}{ Cloning of $\operatorname{tps} C \mathrm{cDNA}$} \\
\hline tpsCReco & ACTCGAATTCCGTCCATGTCAGGGCTCAA & \\
\hline tppAxbaF & ACTGTCTAGAATCCCCCATCATGCT & \multirow{2}{*}{ Cloning of tppA cDNA } \\
\hline tppAecoR & AGTAGAATTCATTAACAGAACCCTCAATAC & \\
\hline tppBxbaF & TACATCTAGATGTCGCCATGACCATCTACA & \multirow{2}{*}{ Cloning of tppB cDNA } \\
\hline tppBkpnR & TTCCGGTACCCCTTTCACTCCTTATCGTGA & \\
\hline tppCFsal & TATCGTCGACCACCCCAATGACGGTCTTCAT & \multirow{2}{*}{ Cloning of tppC cDNA } \\
\hline tppCRbam & CATAGGATCCTCAGTCATGGCTITCTCCGT & \\
\hline
\end{tabular}

reagent (BioRad), gene specific primers (Table 2), and the following protocol: Initial denaturation and enzyme activation, $95^{\circ} \mathrm{C} 30 \mathrm{~s} ; 40$ cycles of $95^{\circ} \mathrm{C}$ for $2 \mathrm{~s}$ and $56-60^{\circ} \mathrm{C}$ for $8 \mathrm{~s}$; plate read; and finally, melt curve analysis starting at $65^{\circ} \mathrm{C}$ and ending at $95^{\circ} \mathrm{C}$. Relative expression for $\operatorname{tps} A-C$ and tppA-C were calculated from and compared to a serially-diluted cDNA pool and normalized to the actinencoding gene (ANI_1_106134), which has been successfully used in previous experiments $[28,31]$ and is expressed at high levels throughout germination according to published microarray data [29]. For each growth stage, the expressions were calculated from four biological replicates, each with three technical replicates. To verify the expression, or lack thereof, in the reconstituted and null mutant of $t p p B$, the expression in mutants was normalized against N402 as previously described [28] using the efficiency calibrated mathematical method for the relative expression ratio in real-time PCR [32].

\section{Gene deletions and complementation}

Deletion constructs for the genes, $t p s A, t p s B, t p p A, t p p B$ and $\operatorname{tpp} C$ were made using fusion PCR to replace the coding sequence with the $A$. oryzae pyrG gene, and used to transform the uridine auxotrophic strain MA70.15 [33] as previously described [29]. With the same technique, a mutant lacking both $t p s B$ and $t p p C$ was created. 
A second deletion mutant of $t p p B,(\triangle t p p B 2)$ was generated in a different uridine auxotrophic strain, MA169.4 [34]. Both MA70.15 and MA169.4 have deficient kusA that is the $A$. niger ortholog of kus70, which is required for the non-homologous end-joining pathway [35]. The tps $C$ deletion strain was constructed by cloning tps $C$ in the standard pBS-SK vector (Stratagene) using BamHI and XhoI. Next, the vector was digested with HindIII to remove $1648 \mathrm{bp}$, containing most of the coding sequence. After dephosphorylation of the vector, a HindIII digested PCR product of the A. oryzae pyrG gene was ligated into the vector, thus replacing $\operatorname{tps} C$. This deletion construct was PCR-amplified and used to transform strain MA169.4. All $A$. niger transformants were confirmed using PCR and sequencing. For the deletion strains where MA169.4 was used as parent strain, the kusA gene was repaired using induced recombination by repeated transfer to agar plates supplemented with fluoroacetamide $0.75 \mu \mathrm{g} / \mathrm{ml}$, as described [34]. All primers for gene deletions are listed in Table 3 . The $\triangle t p p B$ strain was complemented as previously described [28]. Briefly, the strain was transformed with a plasmid carrying an intact copy of $t p p B$ and a cassette carrying hygromycin resistance.

\section{Extraction and quantification of trehalose and trehalose-6-phosphate}

Trehalose from dormant and swollen conidia, germlings and mycelia was extracted and quantified as previously described [28]. In brief, harvested fungal material was freeze-dried and homogenized using a mortar. Samples were diluted with ultra pure water, boiled, evaporated and derivatized by trimethylsilylanization before injection into the gas chromatograph-mass spectrometer (GC-MS). Relative concentrations of $\alpha$ - $\alpha$-trehalose were calculated as the ratio to an internal standard $(\alpha-\beta-$ trehalose) and thereafter correlated to a standard curve to obtain the absolute concentrations. All trehalose measurements were performed in biological duplicates based on the average of three technical triplicates.

Extraction and quantification of T6P was performed essentially as described by [22]. Liquid cultures were inoculated with $10^{6}$ spores per $\mathrm{ml}$, incubated at $25^{\circ} \mathrm{C}$ for 3 days at $140 \mathrm{rpm}$, and all mycelia from one culture made up one sample. Three biological replicates based on the average of three technical replicates were used for all strains.

\section{Stress tolerance and long term viability of conidia}

Dormant conidia from wild-type $A$. niger, the additional control strain pyrG+, and the deletion mutants $\triangle t p p B$ and $\triangle t p p B 2$ were subjected to heat stress for $20,60,90$ and $120 \mathrm{~min}$ at $55^{\circ} \mathrm{C}$. Dormant conidia of wild-type, pyrG + and $\triangle t p p B$ were subjected to sub-lethal salt and benzoic acid stress by being spread on AMM plates containing benzoic acid or $\mathrm{NaCl}$ at concentrations ranging from non-effective to total growth inhibition of the control strains. For detailed description of these stress experiments see [28]. In addition, dormant conidia from control strains and $\triangle t p p B$ were subjected to oxidative stress by adding $200 \mathrm{mM} \mathrm{H}_{2} \mathrm{O}_{2}$ to freshly made conidial suspensions (approximately 250 spores/ml liquid AMM). The suspensions were incubated for 10,20 or $40 \mathrm{~min}$ before being spread on AMM plates. To test long-term viability, conidial suspensions $\left(10^{6}\right.$ conidia/ml water $)$ were stored at $4^{\circ} \mathrm{C}$ for a total of 8 weeks. An aliquot of the suspension was withdrawn weekly, diluted and spread on AMM plates for enumeration.

Plates from all experiments were incubated at $25^{\circ} \mathrm{C}$ for 3-7 days before CFU were estimated, and all experiments were performed at least in triplicates (based on three technical replicates).

\section{Results}

Identification of genes involved in trehalose synthesis in Aspergillus niger and other fungi

Known amino acid sequences of the proteins of the trehalose synthesis complex of $S$. cerevisiae were used as queries to identify homologous genes in the A. niger genome by searching the databases available at NCBI using blastP (http://blast.ncbi.nlm.nih.gov). We confirmed the presence of two previously characterized tps1 orthologs: tpsA (ANI_1_1406074) and tpsB (ANI_1_1078064). Also, we could detect two genes, previously identified by sequence homology [36]; a third tps1 paralog, tpsC (ANI_1_1216124), and a tps2 ortholog, which we call tppA (ANI_1_1432094). In addition, we could identify two previously unidentified, putative tppA paralogs designated tppB (ANI_1_48114) and tppC (ANI_1_2070064). Compared to TppA, these two encoded proteins were of similar length (all three proteins have between 926 to 946 residues) and had a protein identity of $37 \%$ (250 out of 683 ) and 35\% (241 out of 688), respectively (Figure 1). From the NCBI's Conserved Domain Database [37] it was revealed that all three Tpp proteins contain a phosphate synthase domain approximately 200 residues from the N-terminal, and a phosphatase domain approximately 700 residues from the N-terminal (Figure 1). The Tps proteins only contain the phosphate synthase domain (data not shown). In summary, three tps1 orthologs, tpsA-C, and three tps 2 orthologs, tppA-C, were identified from the A. niger genome.

The obtained amino acid sequences of Tpp and Tps proteins were compared to those present in all known genomes of Aspergillus species, as well as other fungal species as references. For this, we used blastP at NCBI (http://blast.ncbi.nlm.nih.gov) and AspND (http://www. aspergillusgenome.org/; [38]; available August 2013). All 
Table 3 Primers used for targeted gene deletions

\begin{tabular}{|c|c|c|}
\hline Primer name & Sequence $5^{\prime}-3^{\prime}$ & Purpose \\
\hline pyrGN2 & CACATGCCTCATTTTGACCA & Mutant confirmation \\
\hline PyrtpsAup & ACCGTTGGAAGGTGGGATCCTATGGATCTCAGAA & \multirow{2}{*}{ Amplifies pyrG with 3' tpsA overhangs } \\
\hline PyrtpsAdown & CCTTTCAGAATGAGTGTGAGCGGATAACAATTTC & \\
\hline tpsAup & CCATCTGTCTAGCTCTTCATCCCC & \multirow{2}{*}{$\operatorname{tps} A$, upstream fragment } \\
\hline tpsApyrup & GATCCATAGGATCCCACCTTCCAACGGTGTAGAGACTCC & \\
\hline tpsApyrdown & TTATCCGCTCACACTCATTCTGAAAGGTGGGGTITC & \multirow{2}{*}{$\operatorname{tps} A$, downstream fragment } \\
\hline tpsAdown & GCAAGATTCCCGCATCCATC & \\
\hline tpsAupN1 & CAACCCCACCAGTTCTCTCAAG & \multirow{2}{*}{ Amplification of KO-fragment } \\
\hline tpsAdownN1 & AAAGGGAGTTCCAAGCAGCCTG & \\
\hline pyrtpsBup* & ATCTGCTCTGCCTGGGATCCTATGGATCTCAGAA & \multirow{2}{*}{ Amplifies pyrG with $3^{\prime}$ tps $B$ overhangs } \\
\hline pyrtpsBdown & CTGCCCATCACCATGTGAGCGGATAACAATTTC & \\
\hline tpsBup* & TTGAACCCTTGAAACCGAACAC & \multirow{2}{*}{ tps $B$, upstream fragment } \\
\hline tpsBpyrGup* & GATCCATAGGATCCCAGGCAGAGCAGATACTTACCCGTC & \\
\hline tpsBpyrGdown & TTATCCGCTCACATGGTGATGGGCAGACGATTG & \multirow{2}{*}{$\operatorname{tps} B$, downstream fragment } \\
\hline tpsBdown & TGCTAAAGAGGGTGTGGGATTG & \\
\hline tpsBupN3 & TCCCGATTGGTAGAATCCCTAAAG & \multirow{2}{*}{ Amplification of tpsB KO-fragment } \\
\hline tpsBdownN3 & CATGCGAAAATGACAGGAACATTC & \\
\hline pyrGuphind & TAAAAGCTTCTATATTGATCCTTA & \multirow{2}{*}{ pyrG, KO of tpsC } \\
\hline pyrGdown & TGTGAGCGGATAACAATTTC & \\
\hline tpsCupN-2 & TGCCGAATTGACGTGCGTAGAG & \multirow{2}{*}{ Cloning of tpsC } \\
\hline tpsCdownN-2 & TGGTGGTGAACCTTTCGTTGTTC & \\
\hline tpsCupN5 & СCСТCCATACTTACTCCATACATCTCG & \multirow{2}{*}{ Amplification of tpsC KO-fragment } \\
\hline tpsCdownN5 & CCAGCTTGACACATCCAACATAAC & \\
\hline pyrtppAup & CCTGTCCCCGCTTCAAGAAAGGGATCCTATGGATCTCAGAA & \multirow{2}{*}{ pyrG with $3^{\prime}$ tppA overhangs } \\
\hline pyrtppAdown & GAGTCATCAGTGCTGCTTTCTGCTGTGAGCGGATAACAATTTC & \\
\hline TppAup & TGTTGGAAGCGTCTITCTGCC & \multirow{2}{*}{ tppA, upstream fragment } \\
\hline tppApyrup & TTCTGAGATCCATAGGATCCCTITCTTGAAGCGGGGACAGG & \\
\hline tppApyrdown & GAAATTGTTATCCGCTCACAGCAGAAAGCAGCACTGATGACTC & \multirow{2}{*}{ tppA, downstream fragment } \\
\hline tppAdown & TGTCCGATTGGGGGTGATTG & \\
\hline tppAupN1 & TGAGGAGGCGTTGTCAAAAGATAG & \multirow{2}{*}{ Amplification of tppA KO-fragment } \\
\hline tppAdownN1 & CGATTGGGGGTGATTGGCTTAC & \\
\hline pyrtppBup & CGGTAGGTTAGGGATCCTATGGATCTCAGAA & \multirow{2}{*}{ Amplification of $A$. oryzae pyrG with $3^{\prime}$ tppB overhangs } \\
\hline pyrtppBdown & GTTTGTCTTGTGTGAGCGGATAACAATTC & \\
\hline tppBup & ATACCAAGCAATCGCCCAAGCCAG & \multirow{2}{*}{ tpp $B$, upstream fragment } \\
\hline tppBpyrGup & TCCATAGGATCCCTAACCTACCGCCCAAAGAGAGAGC & \\
\hline tppBpyrGdown & TTGTTATCCGCTCACACAAGACAAACGATGCGGAATG & \multirow{2}{*}{ tpp $B$, downstream fragment } \\
\hline tppBdown & CGTATCCTGGACTTTCAGCACG & \\
\hline tppBupN1 & TTITCGACCTTGGTGGGTGCTTCC & \multirow{2}{*}{ Amplification of tppB KO-fragment } \\
\hline tppBdownN1 & GAGACATTGTCGGTCAGTGAGGTAG & \\
\hline pyrtppCup & TGTCCTTCAGGGATCCTATGGATCTCAGAA & \multirow{2}{*}{ pyrG with $3^{\prime}$ tppC overhangs } \\
\hline pyrtppCdown* & CTGTTCAGCATTGTGAGCGGATAACAATTTC & \\
\hline tppCup & ATGAGGTGATAGTCGTGGACCCAG & \multirow{2}{*}{ tppC, upstream fragment } \\
\hline tppCpyrup & TCCATAGGATCCCTGAAGGACAAAGACAGGCTGAAG & \\
\hline tppCpyrdown* & TTGTTATCCGCTCACAATGCTGAACAGATGATCCCCAG & tppC, downstream fragment \\
\hline
\end{tabular}


Table 3 Primers used for targeted gene deletions (Continued)

\begin{tabular}{lll}
\hline tppCdown* & TCGAGGTAGAGGTTCCCTTTCG & \\
$\operatorname{tppCupN1*}$ & CGATAGTCTTTGCGAACAGACGG & Amplification of tppC KO-fragment \\
tppCdownN1* & CGAGGTAGAGGTCCCTTTCGATG & Amplification of tpsB/tppC double mutant \\
tpsBupN1 & CCCTTCCCGATTGGTAGAATC & A
\end{tabular}

*Also used in the design of the $\operatorname{tpsB} / \operatorname{tpp} C$ double mutant.

identified fungal genomes contain at least one putative T6P synthase and trehalose-6-phosphate-phosphatases orthologous to Tps $2 / \mathrm{TppA} / \mathrm{Orl}$. In addition, TppB could be identified in all filamentous Ascomycota, whereas TppC is only present in the Aspergillus subgenera Fumigati and Circumdati. Both TppB and TppC group together with the $S$. cerevisiae Tps3 and Tsl1 proteins. The relationships of different gene products in some reference species are displayed as a phylogenetic tree (Figure 2). An additional observation is that, whenever present, $t p s B$ and $t p p C$ are located adjacent on the chromosomes. The protein outside the putative trehalose synthase complex that had the highest blast score against TpsA was ANI_1_512164, encoding a glutamate carboxypeptidase, where the most similar region consisted of $30 \%$ over 50 amino acid residues. In contrast, close homologs could be identified in more distantly related species such as the bacterium Escherichia coli and the protist Dictyostelium discoideum (data not shown).

\section{Two-hybrid assay to reveal putative protein-protein interactions}

In order to determine whether the homologous proteins physically interact, as has been reported in S. cerevisiae [39], we performed a bacterial-based two-hybrid assay screening for interactions between all six $A$. niger proteins. For each protein, the full-length open reading frame was cloned into an expression vector and cotransformed into E. coli cells. All 36 possible combinations of $A$. niger proteins were screened, together with two clones containing different subunits of the leucine zipper GCN4 serving as a positive control and four combinations of one $A$. niger protein and one bacterial protein serving as negative controls. Results with no interactions were repeated at least once in an additional independent two-hybrid assay. Where interactions were detected, the assay was repeated in at least two independent assays. Results indicated that TpsB interacts with TpsA, TpsB and TppA, and that all Tps units interact with themselves (Table 4). All putative interactions involving either $\mathrm{TppB}$ or $\mathrm{TppC}$ did not score any signals above the negative controls (data not shown).

\section{Gene expression during conidial outgrowth}

Gene expressions were quantified during different stages of $A$. niger development. Preliminary results showed that due to the extractability of different structures, two RNA extraction protocols (see Methods) were required: The first included high force to break the tough cell walls of conidia and early germination structures; and, the second was more efficient for fragile structures. Notably, the second protocol was not vigorous enough to extract any RNA from spores (data not shown). Therefore, the "Plate" value in Figure 3 only represents gene expression in mycelia and conidiation structures, but not mRNA present in the produced conidia. cDNA was made with the mRNA as a template, and the relative expressions of the six putative trehalose synthesis genes, $t p s A, t p s B, t p s C, \operatorname{tpp} A, \operatorname{tpp} B$ and $t p p C$, were analyzed with real-time PCR.

The general expression pattern of the genes (Figure 3) was as follows: The expression was highest in still dormant conidia and had decreased by approximately 2 -fold after $3 \mathrm{~h}$ incubation; after $6 \mathrm{~h}$ incubation there was a slight, but not significant, decrease; and, in 12 and $72 \mathrm{~h}$ mycelium the expression was very low. For tpsB, tppA and $\operatorname{tpp} C$, the expression was then up-regulated in sporulating colonies (5 days old), while it remained low for $\operatorname{tps} C$ and $\operatorname{tpp} B$. One gene, tppA, deviated slightly from the described pattern: The decrease in expression after $3 \mathrm{~h}$ was not as profound as in the other genes, and a slight, but not significant, up-regulation could be seen in $72 \mathrm{~h}$ mycelium.

\section{Targeted gene deletions of six Aspergillus niger genes}

To characterize the function of the six $A$. niger proteins, tps $A, t p s B, \operatorname{tps} C, \operatorname{tpp} A, \operatorname{tpp} B$ and $t p p C$ were all subjected to targeted gene deletions by replacing the gene with the A. oryzae pyrG resistance cassette. A double mutant, lacking the two adjacent genes tps $B$ and $\operatorname{tps} C$ was also constructed. All deletion mutants were confirmed with PCR using both internal and flanking primers (data not shown).

With the exception of $\triangle t p p A$, all deletion mutants showed phenotypes similar to wild-type. When culturing the wild-types and mutants at temperatures ranging from $15^{\circ} \mathrm{C}$ to $37^{\circ} \mathrm{C}$, no strain-dependent differences in growth rates or morphologies could be observed; at $10^{\circ} \mathrm{C}$ no growth was observed for any strain (data not shown). The tppA mutant showed a marked reduction in the number of conidia produced compared to the other strains, giving the colonies growing on plate a whitish, and with age, light brownish appearance, compared to the black 


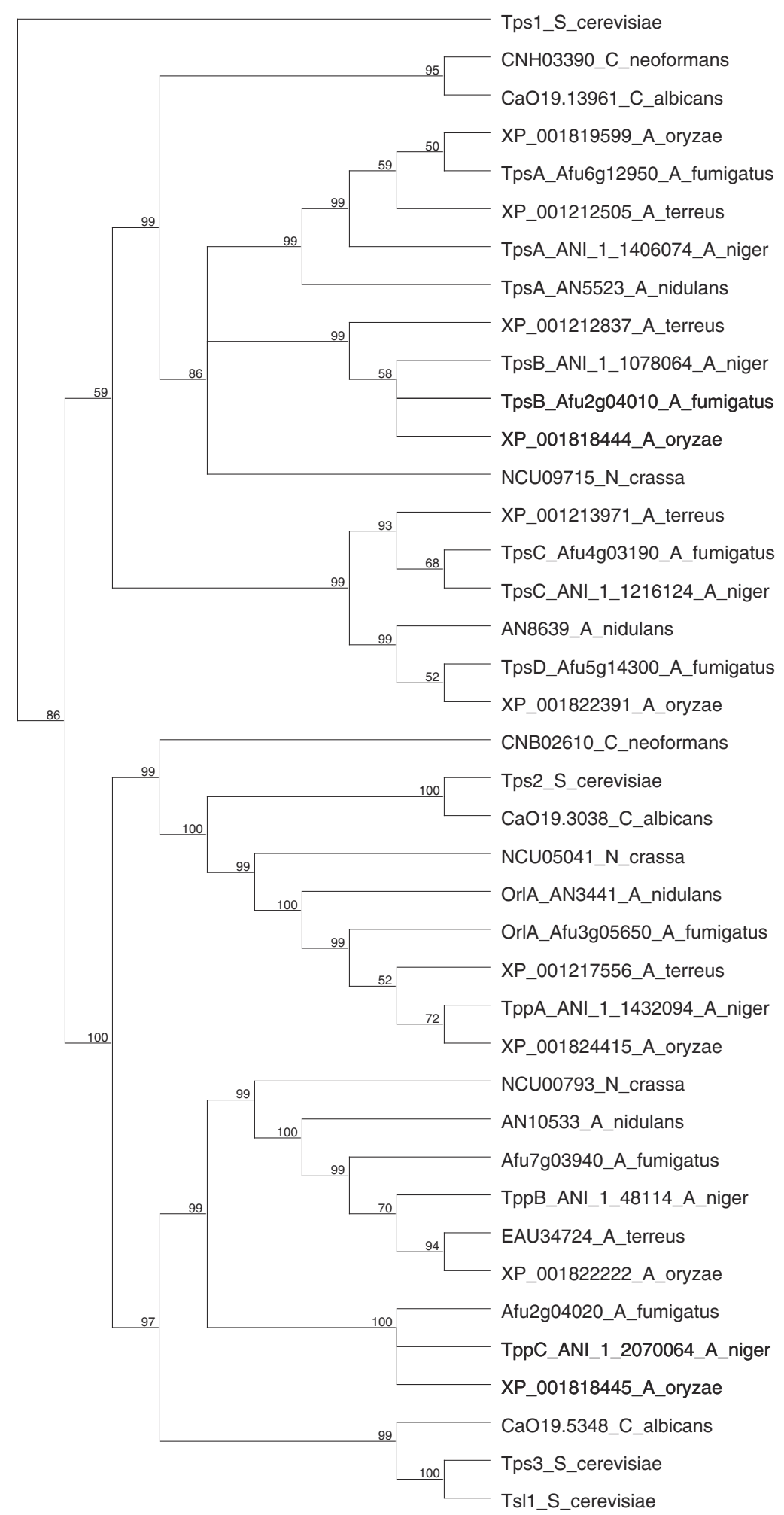

Figure 2 Proteins in the trehalose synthesis family. Analyzed species are: A. fumigatus, A. nidulans, A. niger, A. oryzae, A. terreus, Candida albicans, Cryptococcus neoformans, Neurospora crassa and S. cerevisiae. All proteins from filamentous fungi have their accession number included. For A. niger and previously characterized gene products, given names are also included. This phylogenetic tree was built using the neighbor joining algorithm with 32000 bootstrap replicates. Based on sequence identities, the S. cerevisiae Tps 1 protein was selected by the software as outgroup. Optional settings or use of other algorithms gave identical, or very similar, results. 
Table 4 Protein-protein interactions assayed by Bacterial adenylate cyclase two-hybrid system

\begin{tabular}{lcccc}
\hline Protein & TpsA & TpsB & TpsC & TppA \\
\hline TpsA & $\mathbf{4 1 8}(210-863)^{*}$ & $\mathbf{1 7 4 6}(1582-1799)$ & $113(77-135)$ & 71 (43-89) \\
TpsB & $\mathbf{1 5 9 3 ( 1 4 6 7 - 1 8 3 2 )}$ & $\mathbf{1 7 7 6}(1658-1988)$ & $\mathbf{4 4 1 ( 3 4 1 - 5 6 0 )}$ & $\mathbf{5 8 1}(322-714)$ \\
TpsC & $172(101-244)$ & $\mathbf{6 8 8}(315-980)$ & $\mathbf{1 2 1 4}(861-1551)$ & 80 (67-102) \\
TppA & $429(167-656)$ & $\mathbf{6 9 1}(462-987)$ & $156(133-198)$ & $83(58-98)$ \\
\hline
\end{tabular}

*Estimated values are in units/mg dry weight bacteria. Values in parentheses are the highest and lowest scores for each based on three to four independent assays. The positive control zip-zip (T18 and T25 fragments of the leucine zipper of GCN4) was scored to 3429 (2938-4270). Negative controls and remaining protein interactions scored at maximum 220 (zip-tpsA) but usually less than 50 . Values in bold are considered true protein-protein interactions.

wild-type (Figure 4A,B). This phenotype was retained during aging, and under all growth conditions. When comparing the spore densities, the wild-type, N402, yielded an average of $2.44 \times 10^{6}\left( \pm 0.045 \times 10^{6}\right)$ spores/ $\mathrm{mm}^{2}$, whereas $\triangle t p p A$ yielded an average of $4.40 \times 10^{3}$ $\left( \pm 0.69 \times 10^{3}\right)$ spores $/ \mathrm{mm}^{2}$, i.e. a $6 \times 10^{2}$-fold reduction. Microscopic studies revealed that the conidiophores of $\triangle t p p A$ had a clearly different appearance as is shown in Figure $4 \mathrm{C}$ and D. Most notably, vesicle swelling was almost completely absent and metulae were irregularly positioned (Figure 4C,D and Figure 5). However, the conidia produced showed similar size and ornamentation to wild-type (Figure 5C,F). In contrast to what has been reported in the corresponding mutant of $A$. fumigatus [22], it was not possible to restore wild-type morphology by growing $\triangle t p p A$ on media containing an osmotic stabilizer, i.e. the described phenotype persisted in all growth conditions.

\section{Quantification of trehalose-6-phosphate and trehalose in wild-type and mutants}

All three Tpp genes putatively encode the enzyme trehalose-6-phosphate-phosphatase. To investigate if this enzyme was absent in the Tpp deletion strains, the amount of trehalose-6-phosphate (T6P) in mycelia from wild-type, $\Delta t p p A, \Delta t p p B$ and $\Delta t p p C$ were analyzed. There were no significant differences in T6P levels between wild-type, $\triangle t p p B$ or $\Delta t p p C$. In $\triangle t p p A$, however, T6P was clearly accumulated; the mycelium from this strain contained an average of $124 \mathrm{nmol}$ T6P per gram dry weight compared to $18 \mathrm{nmol}$ in the wild-type (Figure 6).

To elucidate how specific gene products influence the trehalose content of $A$. niger conidia in different stages of maturation, conidia were harvested from control and mutant strains after 5, 14, 28 and 90 days. In these and the following stress experiments, in addition to the wildtype N402 strain, we also included a kusA deficient strain with a repaired pyrG gene, pyrG $+[28]$ as a control with identical genetic background as the tps and tpp deletion mutants. The dormant conidia were extracted and the trehalose levels analyzed and expressed as percentage of conidial dry weight (Figure 7). For $\triangle t p p A$ it was not possible to analyze the trehalose content of 5 day conidia, as insufficient conidia were produced. For the other strains, a significant increase in trehalose was detected between the two first time points tested, 5 and 14 days. During further aging of conidia, between 14
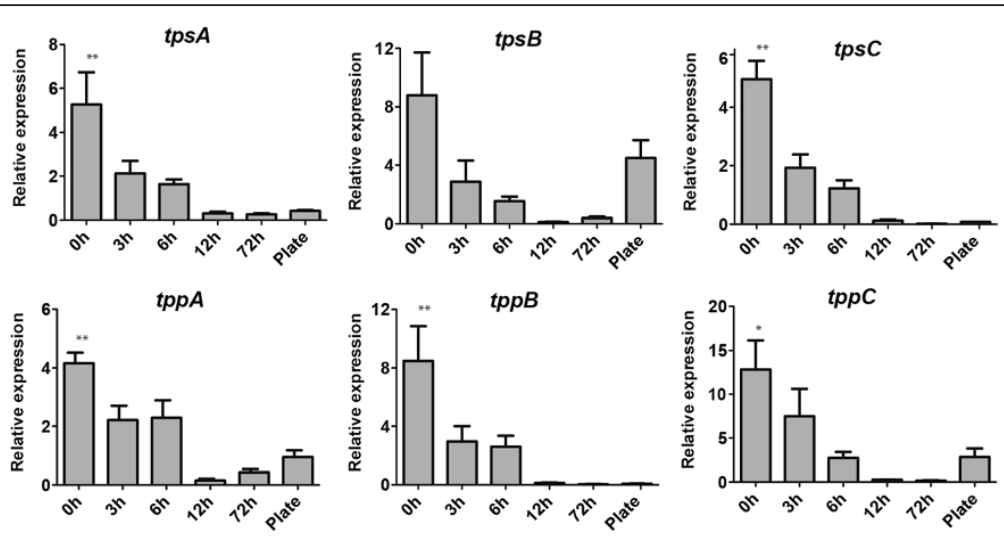

Figure 3 Expression of putative trehalose synthesis genes during outgrowth of $\boldsymbol{A}$. niger conidia. The developmental stages are given on the $x$ - axis: 0 h are dormant conidia; 3-72 h are swollen conidia, germlings or mycelia after so many hours of incubation in liquid AMM media; and Plate is the entire sporulating culture grown on AMM plates for 5 days. Error bars show standard error of the mean based on four biological replicates each calculated as the average of three technical replicates. For all genes, the expressions are normalized against the expression of actin. ${ }^{*}$ Indicates that the expression at $0 \mathrm{~h}$ was statistically significant to the following time-points within the same group except $3 \mathrm{~h}$ (one-way ANOVA, $P<0.05$ ). ${ }^{*}$ Indicates that the expressions at $0 \mathrm{~h}$ were statistically significant to all of the following time-points within the same group (one-way ANOVA, $P<0.05$ ). 

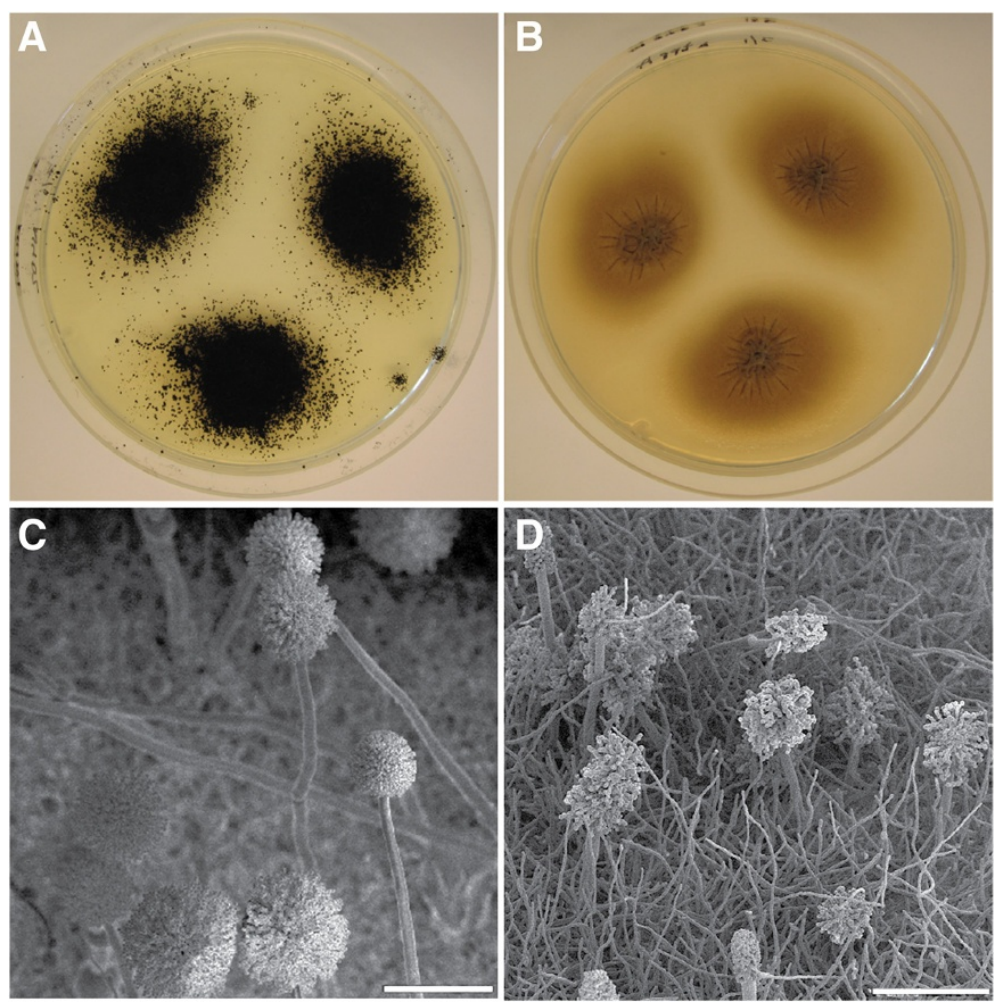

Figure 4 Morphologies of cultures grown for 1 week on AMM. Wild-type, left (A and C), and $\triangle$ tppA right (B and D). Size bars of SEM photos are $100 \mu \mathrm{m}$

and 90 days, no significant changes in trehalose levels were detected (with the exception of $\Delta t p s B-\Delta t p p C$ where the level in 28 day old conidia was $4.6 \%$ compared to 3.5 and 3.8 in 14 and 90 day old conidia, respectively).

At all time points, conidia from all mutant strains contained significantly less trehalose compared to wild-type conidia (again, with the exception of $\Delta t p s B-\Delta t p p C 28$ days). When comparing the deletion mutants to the other control strain, $p y r G+$, significantly lower levels of trehalose were detected in strains $\triangle t p s A, \triangle t p p A$ and $\triangle t p p B$. After 14 days of maturation the conidial trehalose level was $50 \%$ lower in $\triangle t p s A$ compared to $p y r G+$, and 73 and $60 \%$ lower in $\triangle t p p A$


Figure 5 Detailed morphologies of cultures grown for 1 week on AMM. Wild-type, top (A, B and C), and $\triangle$ tppA bottom (D, E and F). Size bars: $\mathbf{A}=20 \mu \mathrm{m}, \mathbf{B}=10 \mu \mathrm{m}, \mathbf{C}=10 \mu \mathrm{m}, \mathbf{D}=10 \mu \mathrm{m}, \mathbf{E}=10 \mu \mathrm{m}, \mathbf{F}=5 \mu \mathrm{m}$. 




Figure 6 Content of T6P in mycelium dry weight of wild-type and Tpp deletion mutants. Error bars show standard error of the mean. In $\Delta$ tppA, the level of T6P was significantly higher compared to all other strains (one-way ANOVA, $P<0.05$ )

and $\triangle t p p B$, respectively. For $\triangle t p s A$ and $\triangle t p p A$, the reduction was significant at all time points tested, and for $\triangle t p p B$, the difference was significant in 14, 28 and 90 day old conidia but not after 5 days.

Among the deletion mutants with wild-type like phenotypes, i.e. when excluding $\triangle t p p A, \triangle t p p B$ had the lowest overall trehalose content. After 14 days of incubation, the trehalose level was $1.7 \%$ of conidial dry weight compared to 5.1 and $4.1 \%$ in wild-type $\mathrm{N} 402$ and $p y r G+$, respectively. Although the conidial trehalose content was consistently lower in $\triangle t p p A$, the extremely low number of spores produced made this strain unsuitable for studies on conidial survival. Therefore, $\triangle t p p B$ was, due to its wildtype morphology, selected for additional studies to reveal whether or not a normal internal trehalose level has any impact on stress survival and growth.

\section{Confirmation and further characterization of $\triangle t p p B$}

Before subjecting the tppB deletion mutant to stress, a few confirmatory experiments were performed to ensure that the lowered trehalose content was a consequence of the deleted gene: A new deletion mutant of $\operatorname{tpp} B, \triangle t p p B 2$, was generated using MA169.4 as parent strain, and on a selected transformant the $\Delta k u s A$ gene was restored using acetamide. Analysis of trehalose content in 14 day old conidia from this new mutant showed that they were as low as in $\triangle t p p B(1.54 \pm 0.1 \%$ of conidia dry weight in $\triangle t p p B 2$ versus $1.72 \pm 0.5 \%$ in $\triangle t p p B)$. Moreover, the deletions mutants were complemented by transformation of an autonomously replicating plasmid carrying the gene for hygromycin resistance as well as an intact copy of the tppB gene. Putative transformants were selected on hygromycin plates. The presence of the construct was confirmed using PCR and plasmid rescue (data not shown). In a previous study we discovered that, when using this methodology, only a fraction of conidia carry the plasmid [28]. This was also valid for tpp $B$ + conidia, where only a few percent germinated on hygromycin media (data not shown). Therefore, the mycelial trehalose content was measured after growth in liquid AMM media supplemented with hygromycin under a continuous selection pressure to retain the plasmid, we showed that in $t p p B$ + the amount of trehalose was restored to wild-type level (Figure $8 \mathrm{~A}$ ). The expression of $\operatorname{tpp} B$ was examined in mycelium from wild-type, $\triangle t p p B$ and $t p p B+$. In the deletion mutant, no expression was detected, whereas in the complemented strain, the levels were in the same range as in the wildtype (Figure 8B). From these experiments we concluded that the deletion of $\operatorname{tpp} B$ causes the lowered trehalose levels in $\triangle t p p B$. However, since the plasmid carrying the wild-type version of the gene was lost in most conidia, the $\operatorname{tpp} B+$ strain was not included in the following experiments.

To evaluate the importance of trehalose as a stress protectant, the trehalose contents of the $\triangle t p p B$ mutant and the control strains were analyzed in early stages of germination, and were subjected to lethal and sub-lethal heat and oxidative stress as well as sub-lethal salt and acid stress. The trehalose levels in $\triangle t p p B$ followed the same pattern of breakdown and re-synthesis as in the control strains, but they were consistently lower in accordance with the lower initial value (Figure 9). Dormant conidia of $\triangle t p p B$ were significantly less tolerant to heat stress compared to the control strains; After 60 min of heat stress, the survival of $\triangle t p p B$ was $35 \%$ compared to $78 \%$ in



Figure 7 Trehalose content in mutant and wild-type conidia of different age. The numbers to the right represent how many days the colony had grown on AMM plates before conidia were harvested and analysed. Error bars show standard error of the mean. 

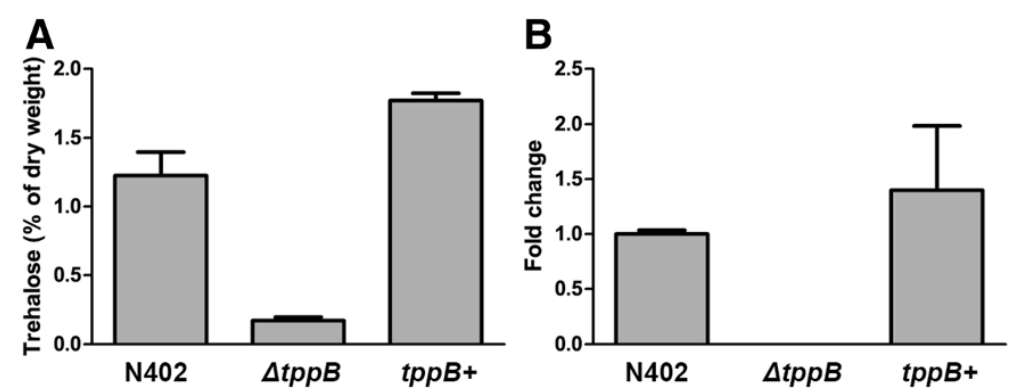

Figure 8 Trehalose content of mycelium (A) and relative expression of tppB (B). Error bars show standard error of the mean, based on three biological replicates, and for qPCR each biological replicate was calculated as the average of three technical replicates.

wild-type. After an additional $60 \mathrm{~min}$, the survival of $\triangle t p p B$ further decreased to $2 \%$ compared to $38 \%$ in wild-type. (Figure 10). These experiments were repeated with the new independent deletion mutant, $\triangle t p p B 2$, and the results were identical to those for $\triangle t p p B$ (data not shown). For the other stressors tested, benzoic acid, $\mathrm{NaCl}$ and $\mathrm{H}_{2} \mathrm{O}_{2}$, as well as long-term viability where conidia were stored in water at $4^{\circ} \mathrm{C}$ for a total of 8 weeks, no significant differences between the mutant and the control strains could be detected (data not shown).

\section{Discussion}

In this project we have studied six genes with a putative role in trehalose synthesis in $A$. niger: $\operatorname{tps} A, \operatorname{tps} B, \operatorname{tps} C$, tpp $A, t p p B$ and tppC. All six genes encode homologous proteins and no similar gene products within the $A$. niger genome could be detected. Three proteins, TpsA, $\mathrm{TpsB}$ and $\mathrm{TpsC}$, have previously been identified as orthologs to the yeast protein Tps1. As the orthologs are conserved in related species, it is plausible that there is a

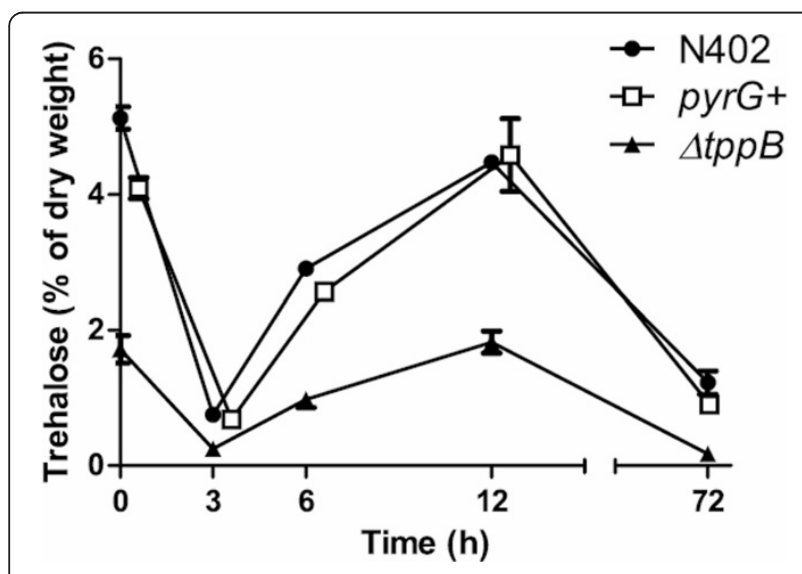

Figure 9 Concentration of trehalose during outgrowth of wildtype, pyrG + and $\Delta$ tpp B conidia. Note the scale break between 12 and $72 \mathrm{~h}$ and that pyrG + observations are horizontally offset to avoid visual overlap. The error bars represent the standard error of the mean. The level of trehalose in $\triangle \operatorname{tp} p B$ was significantly different compared to wild-type for all time points except $3 \mathrm{~h}$ (two-way ANOVA, $P<0.0001$ at 0 , 6 and $12 \mathrm{~h}$, and $P<0.01$ at $72 \mathrm{~h}$ ) functional differentiation between the paralogs, e.g. one paralog could be essential for trehalose synthesis in conidia, whereas another paralog is strictly induced by stress. This assumption is in line with the previous observation in $A$. niger where the expression of tps $B$ is stress-induced whereas tps $A$ is constitutively expressed [23], although our data also suggest that $\operatorname{tps} B$ has a role during differentiation (see Figure 3). When deleting the trehalose-phosphate-synthase paralogs, only $\Delta t p s A$ displayed a reduced trehalose content. The lower level in this mutant is in line with a previous report using a different target strain and deletion procedure [23]. In the related fungus, A. fumigatus, a tpsA/tps $B$ double deletion resulted in a strain with depleted trehalose content, and in the same study, it was shown that the expressions of $\operatorname{tps} C$ and $-D$ were very low at all time points [12]. These authors evaluated their expression

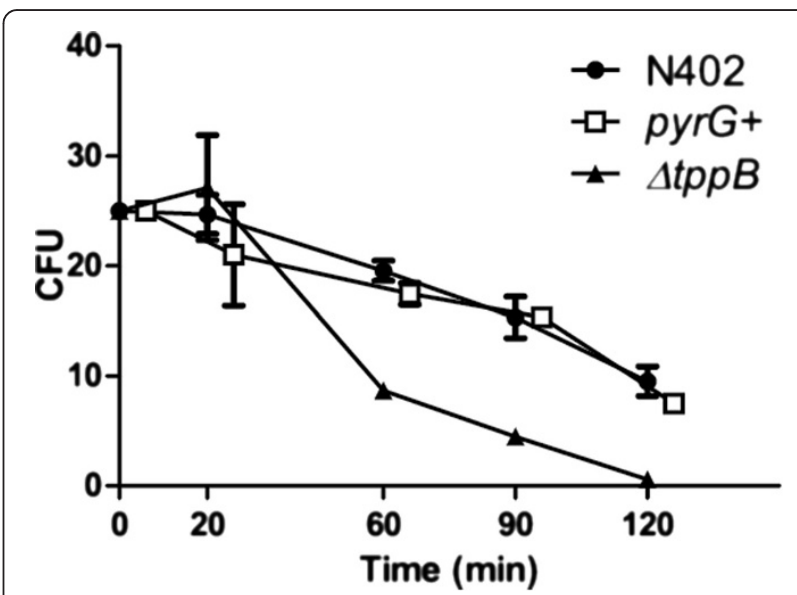

Figure 10 Viabilities of dormant $A$. niger conidia after subjection to heat stress. Conidia were held at $55^{\circ} \mathrm{C}$ for $20,60,90$ and $120 \mathrm{~min}$. For all strains, the numbers of counted colonies were normalized to 25 at time $=0$ min to avoid differences in numbers of assayed spores. Note that pyrG + observations are horizontally offset to avoid visual overlap. There were no significant differences between the control strains (N402 and pyrG+). Except for the two first time points, the viability of $\triangle t p p B$ was significantly lower than in N402 (two-way ANOVA, $P<0.01$ in 60 and $90 \mathrm{~min}$, and $P<0.05$ in $120 \mathrm{~min}$ ). 
data using RNA extracted from hyphae, and in the present study, the $A$. niger tps $C$ was expressed at very low levels at $72 \mathrm{~h}$. Thus the results from the two fungi are not contradictory, and most likely an $A$. niger tps $A / t p s B$ deletion mutant would also have a depleted trehalose content. The results from $A$. niger and A. fumigatus are also in accordance with findings in $A$. nidulans where deletion of tpsA resulted in depleted trehalose content [11], as that species does not have the tpsB paralogue. A conclusion from studying the trehalose content from these three species is that TpsA is the most important trehalosephosphate-synthase under normal conditions, but lack of the tpsA gene can be fully compensated by TpsB in A. fumigatus and partly by at least one of TpsB or TpsC in $A$. niger, but not by TpsD in $A$. nidulans.

The deletion mutant with the most distinctive characteristics in our experiments was $\triangle t p p A$, i.e. with an abnormal morphology and reduced levels of both trehalose-6phosphate and trehalose. The altered morphology of the strain is probably due to toxicity of T6P as indicated for the corresponding deletion mutant in A. fumigatus [22]. However, in $A$. niger as well as $A$. fumigatus and $A$. nidulans [12,25], mutants of tppA are not totally lacking in trehalose. Therefore, it is possible that under specific conditions, e.g. when TppA is absent, TppB, and also $\mathrm{TppC}$ where present, may contribute to some T6P activity. Another possibility is that the sugar can be synthesized by proteins other than Tps/Tpp, e.g. the Trehalose Phosphorylase pathway, for which putative genes have been identified and partially characterized in $N$. crassa [40] and A. fumigatus [22] and also exist in A. niger (ANI_1_2720024). However, it is possible to generate mutants, within the homologous Tps/Tpp group, in A. fumigatus and A. nidulans that totally lack trehalose $[11,12]$. Therefore, we believe that this is the only active trehalose synthesis pathway in Aspergilli. However, internal trehalose contents may not solely be dependent on the presence and expression of these six genes, as in S. cerevisiae there is a strong linkage between trehalose synthesis and the degrading trehalases [41] as well as evidences of posttranscriptional activation of the genes involved in trehalose metabolism $[42,43]$.

Besides a putative phosphatase activity, TppB and $\mathrm{TppC}$ may have similar biological roles as the yeast proteins Tps3 and Tsl1, which also contain phosphatase domains - in yeasts, deletion of both genes is necessary before some reduction in internal trehalose content can be observed [17]. It is intriguing that $\operatorname{tps} B$ and $\operatorname{tpp} C$ are linked on the chromosome. We cannot explain why the conidial trehalose content in this double mutant was significantly higher after 28 days, but based on the expression patterns (see Figure 3), it is possible that the expression of the two genes are regulated by the same factors. In addition to the above-mentioned observations, some conclusions can be drawn from the gene expression data: All identified genes were expressed, indicating that the paralogs are not inactive duplicates. For tps $C$ and $\operatorname{tpp} B$, the expressions were consistently low after $6 \mathrm{~h}$, indicating that the two genes may be regulated by the same mechanism. This assumption is supported by a previous observation using $A$. oryzae arrays where the tps $C$ and $t p p B$ orthologs were down-regulated in a deletion strain of atfA, a gene encoding a transcription factor [44]. To our knowledge, two previous studies describing the expression of trehalose synthesis genes in A. niger during germination, using microarray technology, or in combination with RNA sequencing, have been published $[29,45]$. With the exception that van Leeuwen and co-workers [29] saw a drastic drop after $2 \mathrm{~h}$ and then a gradual up-regulation of $\operatorname{tps} A$ and $\operatorname{tps} B$, those results are in line with our findings.

The extensive measurements of internal trehalose indicate that the trehalose contents, for all strains, were low in 5 day old conidia, significantly elevated in 14 day old conidia, and then maintained at the value of 14 days (Figure 7). A plausible hypothesis is that conidia of $A$. niger reach full maturity, at least in terms of trehalose accumulation, sometime between 5 days and 2 weeks. Consequently it is not advisable to perform stress experiments on young conidia because their trehalose content is not necessarily typical for the final level, especially not in kusA deficient strains that seem to have slower conidial maturation in terms of trehalose content.

We found that 2 week old conidia of $\triangle t p p B$ were more susceptible to heat shock than wild-type conidia, indicating that trehalose protects the spores from thermal stress. These results are in line with earlier studies in Aspergillus species $[11,12,23]$. However, in contrast to results from $A$. fumigatus and $A$. nidulans, we could not detect any increased sensitivity of $\triangle t p p B$ to oxidative stress $[11,12]$, salt or acid stress, or any decreased viability after long term storage. It should be noted that unlike $\triangle t p p B$ in our experiments, which harbored approximately one third of wild-type trehalose content, the $A$. fumigatus and $A$. nidulans mutants were totally depleted of trehalose.

In $S$. cerevisiae it has been shown that, using a twohybrid assay, the four homologous proteins physically interact. When repeating the experiments using the six identified $A$. niger proteins, we could observe interactions for four of six proteins. These results suggest that TppA and TpsA-C form a complex, while the phylogenetically more distant proteins, $\mathrm{TppB}$ and $\mathrm{TppC}$, are present outside the complex. However, due to the experimental limits, it is possible that neither TppB nor $\mathrm{TppC}$ was correctly folded and therefore not interacting. It is notable that in S. cerevisiae, a truncated version of Tsl1 was necessary for the success of the 
interaction experiments [40], in contrast to our experiment in which we only used full-length proteins.

\section{Conclusions}

To conclude, in this study novel information about the six gene products involved in trehalose synthesis in $A$. niger has been generated. When characterizing deletion mutants, lack of the most conserved trehalose phosphate synthase tpsA, the trehalose phosphate phosphatase tpp $A$, or the previously non-characterized tpp $B$, resulted in lower trehalose contents. An additional insight is that the components in a putative trehalose synthesis complex differ among the Aspergilli, but some gene products are common throughout the fungal kingdom.

\section{Competing interests}

The authors declare the absence of competing interests.

\section{Authors' contributions}

Ås performed the majority of the laboratorial work. Ås and PM performed all experiments with exception of the RNA extraction from dormant conidia and conidia in early stages of germination, performed by MRL, and the SEM studies, performed by JD. Ås and PM conceived and designed the study and wrote the manuscript. All authors read and approved the final manuscript

\section{Acknowledgements}

Dr. Jonathan Hilmer for assistance with the T6P analysis and Dr. Su-lin Leong for proofreading the manuscript before submission, are greatly acknowledged. This work was financed by the Swedish research council Formas.

\section{Author details}

'Uppsala BioCenter, Department of Microbiology, Swedish University of Agricultural Sciences, P.O. Box 7025, SE-750 07 Uppsala, Sweden. ${ }^{2}$ Applied and Industrial Mycology, CBS-KNAW Fungal Biodiversity Centre, Uppsalalaan 8, 3584 CT Utrecht, The Netherlands. ${ }^{3}$ Present address: Swedish Chemicals Agency, Box 2, SE-172 13 Sundbyberg, Sweden.

Received: 23 December 2013 Accepted: 8 April 2014

Published: 11 April 2014

\section{References}

1. Avonce N, Mendoza-Vargas A, Morett E, Iturriaga G: Insights on the evolution of trehalose biosynthesis. BMC Evol Biol 2006, 6:109.

2. Iordachescu M, Imai R: Trehalose biosynthesis in response to abiotic stresses. J Integr Plant Biol 2008, 50(10):1223-1229.

3. Elbein AD, Pan YT, Pastuszak I, Carroll D: New insights on trehalose: a multifunctional molecule. Glycobiology 2003, 13(4):17R-27R.

4. Thevelein JM: Regulation of trehalose mobilization in fungi. Microbiol Mol Biol Rev 1984, 48(1):42-59.

5. Elbein AD: The metabolism of a, a-trehalose. Adv Carbohydr Chem Biochem 1974, 30:227-256.

6. Gancedo C, Flores CL: The importance of a functional trehalose biosynthetic pathway for the life of yeasts and fungi. FEMS Yeast Res 2004, 4(4-5):351-359.

7. Crowe JH, Hoekstra FA, Crowe LM: Anhydrobiosis. Annu Rev Physiol 1992, 54:579-599.

8. Wiemken A: Trehalose in yeast, stress protectant rather than reserve carbohydrate. Antonie Van Leeuwenhoek 1990, 58(3):209-217.

9. Hottiger T, Virgilio C, Hall M, Boller T, Wiemken A: The role of trehalose synthesis for the acquisition of thermotolerance in yeast. Eur J Biochem 1994, 219(1-2):187-193.

10. Cheng L, Moghraby J, Piper PW: Weak organic acid treatment causes a trehalose accumulation in low-pH cultures of Saccharomyces cerevisiae, not displayed by the more preservative-resistant Zygosaccharomyces bailii. FEMS Microbiol Lett 1999, 170(1):89-95.

11. Fillinger $S$, Chaveroche M-K, van Dijck P, de Vries R, Ruijter G, Thevelein J, $d^{\prime}$ Enfert C: Trehalose is required for the acquisition of tolerance to a variety of stresses in the filamentous fungus Aspergillus nidulans. Microbiology 2001, 147(7):1851-1862.

12. Al-Bader N, Vanier G, Liu H, Gravelat FN, Urb M, Hoareau CMQ, Campoli P, Chabot J, Filler SG, Sheppard DC: Role of trehalose biosynthesis in Aspergillus fumigatus development, stress response, and virulence. Infect Immun 2010, 78(7):3007-3018.

13. Uyar EO, Hamamci $H$, Turkel $S$ : Effect of different stresses on trehalose levels in Rhizopus oryzae. J Basic Microbiol 2010, 50(4):368-372.

14. Doehlemann G, Berndt P, Hahn M: Trehalose metabolism is important for heat stress tolerance and spore germination of Botrytis cinerea. Microbiol-Sgm 2006, 152:2625-2634.

15. Jain NK, Roy I: Effect of trehalose on protein structure. Protein Sci 2009, 18(1):24-36

16. Lins RD, Pereira CS, Hünenberger PH: Trehalose-protein interaction in aqueous solution. Proteins Struct Funct Bioinf 2004, 55(1):177-186.

17. Bell W, Sun WN, Hohmann S, Wera S, Reinders A, De Virgilio C, Wiemken A, Thevelein JM: Composition and functional analysis of the Saccharomyces cerevisiae trehalose synthase complex. J Biol Chem 1998, 273(50):33311-33319.

18. de Virgilio C, Burckert N, Bell W, Jeno P, Boller T, Wiemken A: Disruption of Tps2, the gene encoding the 100-kDa subunit of the trehalose-6phosphate synthase phosphatase complex in Saccharomyces cerevisiae, causes accumulation of trehalose-6-phosphate and loss of trehalose-6phopshate phosphatase activity. Eur J Biochem 1993, 212(2):315-323.

19. Londesborough J, Vuorio O: Trehalose-6-phosphate synthase/phosphatase complex from bakers' yeast: purification of a proteolytically activated form. J Gen Microbiol 1991, 137(2):323-330.

20. d'Enfert C: Fungal spore germination: insights from the molecular genetics of Aspergillus nidulans and Neurospora crassa. Fungal Genet Biol 1997, 21(2):163-172.

21. Foster AJ, Jenkinson JM, Talbot NJ: Trehalose synthesis and metabolism are required at different stages of plant infection by Magnaporthe grisea. EMBO J 2003, 22(2):225-235.

22. Puttikamonkul S, Willger SD, Grahl N, Perfect JR, Movahed N, Bothner B, Park S, Paderu P, Perlin DS, Cramer RA Jr: Trehalose 6-phosphate phosphatase is required for cell wall integrity and fungal virulence but not trehalose biosynthesis in the human fungal pathogen Aspergillus fumigatus. Mol Microbiol 2010, 77(4):891-911.

23. Wolschek MF, Kubicek CP: The filamentous fungus Aspergillus niger contains two "differentially regulated" trehalose-6-phosphate synthaseencoding genes, tpsA and tpsB. J Biol Chem 1997, 272(5):2729-2735.

24. Thevelein JM, Hohmann S: Trehalose synthase - guard to the gate of glycolysis in yeast. Trends Biochem Sci 1995, 20(1):3-10.

25. Borgia PT, Miao YH, Dodge CL: The orlA gene from Aspergillus nidulans encodes a trehalose-6-phosphate phosphatase necessary for normal growth and chitin synthesis at elevated temperatures. Mol Microbiol 1996, 20(6):1287-1296.

26. Schuster E, Dunn-Coleman N, Frisvald JC, van Dijck PW: On the safety of Aspergillus niger-a review. Appl Microbiol Biotech 2002, 59:426-435.

27. Bos CJ, Debets AJM, Swart K, Huybers A, Kobus G, Slakhorst SM: Geneticanalysis and the construction of master strains for assignment of genes to 6 linkage groups in Aspergillus niger. Curr Genet 1988, 14(5):437-443.

28. Svanström $\AA$, Melin P: Intracellular trehalase activity is required for development, germination and heat-stress resistance of Aspergillus niger conidia. Res Microbiol 2013, 164(2):91-99.

29. van Leeuwen MR, Krijgsheld P, Bleichrodt R, Menke H, Stam H, Stark J, Wosten HAB, Dijksterhuis J: Germination of conidia of Aspergillus niger is accompanied by major changes in RNA profiles. Stud Mycol 2013, 74:59-70.

30. Plumridge A, Melin P, Stratford M, Novodvorska M, Shunburne L, Dyer PS, Roubos JA, Menke H, Stark J, Stam H, Archer DB: The decarboxylation of the weak-acid preservative, sorbic acid, is encoded by linked genes in Aspergillus spp. Fungal Genet Biol 2010, 47(8):683-692.

31. Bohle K, Junglebloud A, Göcke Y, Dalpiaz A, Cordes C, Horn H, Hempel DC Selection of reference genes for normalisation of specific gene quantification data of Aspergillus niger. J Biotech 2007, 132:353-358.

32. Pfaffl MW: A new mathematical model for relative quantification in real-time RT-PCR. Nucleic Acids Res 2001, 29(9):e45.

33. Meyer V, Arentshorst M, El-Ghezal A, Drews A-C, Kooistra R, van den Hondel CAMJJ, Ram AFJ: Highly efficient gene targeting in the Aspergillus niger kusA mutant. J Biotechnol 2007, 128:770-775. 
34. Carvalho N, Arentshorst M, Kwon MJ, Meyer V, Ram AFJ: Expanding the ku70 toolbox for filamentous fungi: establishment of complementation vectors and recipient strains for advanced gene analyses. Appl Microbio/ Biotechnol 2010, 87(4):1463-1473.

35. Dudasova Z, Dudas A, Chovanec M: Non-homologous end-joining factors of Saccharomyces cerevisiae. FEMS Microbiol Rev 2004, 28:581-601.

36. Pel HJ, de Winde JH, Archer DB, Dyer PS, Hofmann G, Schaap PJ, Turner G, de Vries RP, Albang R, Albermann $K$, Andersen MR, Bendtsen JD, Benen JAE, van den Berg M, Breestraat S, Caddick MX, Contreras R, Cornell M, Coutinho PM, Danchin EGJ, Debets AJM, Dekker P, van Dijck A, Dijkhuizen L, Driessen AJM, d'Enfert C, Geysens S, Goosen C, Groot GSP: Genome sequencing and analysis of the versatile cell factory Aspergillus niger CBS 513.88. Nat Biotechnol 2007, 25(2):221-231.

37. Marchler-Bauer A, Lu S, Anderson JB, Chitsaz F, Derbyshire MK, DeWeese-Scott C, Fong JH, Geer LY, Geer RC, Gonzales NR, Gwadz M, Hurwitz DI, Jackson JD, Ke Z, Lanczycki CJ, Lu F, Marchler GH, Mullokandov M, Omelchenko MV, Robertson CL, Song JS, Thanki N, Yamashita RA, Zhang D, Zhang N, Zheng C, Bryant SH: CDD a Conserved Domain Database for the functional annotation of proteins. Nucleic Acids Res 2011, 39:D225-D229.

38. Arnaud MB, Cerqueira GC, Inglis DO, Skrzypek MS, Binkley J, Chibucos MC, Crabtree J, Howarth C, Orvis J, Shah P, Wymore F, Binkley G, Miyasato SR, Simison M, Sherlock G, Wortman JR: The Aspergillus Genome Database (AspGD): recent developments in comprehensive multispecies curation, comparative genomics and community resources. Nucleic Acids Res 2012, 40(D1):D653-D659.

39. Reinders A, Bürckert N, Hohmann S, Thevelein JM, Boller T, Wiemken A, De Virgilio C: Structural analysis of the subunits of the trehalose-6-phosphate synthase/phosphatase complex in Saccharomyces cerevisiae and their function during heat shock. Mol Microbiol 1997, 24(4):687-696.

40. Shinohara ML, Correa A, Bell-Pedersen D, Dunlap JC, Loros JJ: Neurospora Clock-Controlled Gene 9 (ccg-9) encodes trehalose synthase: circadian regulation of stress responses and development. Eukaryot Cell 2002, 1(1):33-43.

41. Jules M, Beltran G, Francois J, Parrou JL: New insights into trehalose metabolism by Saccharomyces cerevisiae: NTH2 encodes a functional cytosolic trehalase, and deletion of TPS1 reveals Ath1p-dependent trehalose mobilization. App/ Environ Microbio/ 2008, 74(3):605-614.

42. Hirimburegama K, Durnez P, Keleman J, Oris E, Vergauwen R, Mergelsberg H, Thevelein JM: Nutrient-induced activation of trehalose in nutrient-starved cells of the yeast Saccharomyces cerevisiae: CAMP is not involved as second messenger. J Gen Microbiol 1992, 138:2035-2043.

43. Giots F, Donaton MCV, Thevelein JM: Inorganic phosphate is sensed by specific phosphate carriers and acts in concert with glucose as a nutrient signal for activation of the protein kinase A pathway in the yeast Saccharomyces cerevisiae. Mol Microbiol 2003, 47(4):1163-1181.

44. Sakamoto K, Iwashita K, Yamada O, Kobayashi K, Mizuno A, Akita O, Mikami $\mathrm{S}$, Shimoi H, Gomi K: Aspergillus oryzae atfA controls conidial germination and stress tolerance. Fungal Genet Biol 2009, 46(12):887-897.

45. Novodvorska M, Hayer K, Pullan ST, Wilson R, Blythe MJ, Stam H, Stratford M, Archer DB: Trancriptional landscape of Aspergillus niger at breaking of conidial dormancy revealed by RNA-sequencing. BMC Genomics 2013, $14: 246$.

doi:10.1186/1471-2180-14-90

Cite this article as: Svanström et al:: Trehalose synthesis in Aspergillus niger: characterization of six homologous genes, all with conserved orthologs in related species. BMC Microbiology 2014 14:90.

\section{Submit your next manuscript to BioMed Central and take full advantage of:}

- Convenient online submission

- Thorough peer review

- No space constraints or color figure charges

- Immediate publication on acceptance

- Inclusion in PubMed, CAS, Scopus and Google Scholar

- Research which is freely available for redistribution

Submit your manuscript at www.biomedcentral.com/submit
C) Biomed Central 